\title{
Numerical analysis of effects of adhesive type and geometry on mixed-mode failure of adhesive joint
}

\author{
Lijuan Liao*, Chenguang Huang \\ Key Laboratory for Mechanics in Fluid Solid Coupling Systems, Institute of Mechanics, Chinese Academy of Sciences, Beijing, 100190, \\ People's Republic of China
}

\section{A R T I C L E I N F O}

Keywords:

Mixed-mode failure

Cohesive parameters

Cohesive zone model (CZM)

Double scarf adhesive joint (DSJ)

\begin{abstract}
A B S T R A C T
In the present study, the effects of the adhesive type and geometry (adhesive thickness and scarf angle) on mixed-mode failure of double scarf adhesive joint (DSJ) under uniaxial tensile loading were numerically examined using the finite element subroutine which coupled with a mixed-mode cohesive zone model (CZM). Especially, the effects of the adhesive type, which actually represent the influences of the cohesive parameters in mode I and mode II, on the mechanical properties of DSJ were discussed systematically. The numerical results reveal that the ultimate tensile loading and the necessary energy for failure of DSJ are controlled by the intrinsic components in mode I and mode II with different rates. Accordingly, the mathematical expressions for the ultimate tensile loading and the failure energy of DSJ with respect to the thickness-dependency cohesive parameters in two modes (I and II) and the scarf angle were deduced to identify each contribution in each mode component for a given type of adhesive. In addition, the numerical results also demonstrate that relationship between the interface damage level (corresponding to the ultimate tensile loading) and the adhesive thickness is not monotonous. However, as an increase of the adhesive thickness, the uniformity of damage level distribution is enhanced. Furthermore, the variation of the interface damage level with respect to the scarf angle is also not monotonous for each adhesive thickness. It can be concluded that the effects of the scarf angle and the adhesive thickness on the mixed-mode failure of DSJ are coupled rather than independently.
\end{abstract}

(c) 2015 Elsevier Ltd. All rights reserved.

\section{Introduction}

As an ideal alternative joining method compared with the conventional mechanical fastening techniques, adhesive joint, due to its merits such as lightweight and high strength, has been widely used in industries. Consequently, the failure mechanism examination plays a crucial role in the design aiming to improve the safety and economy of adhesive structures. Thus, in order to promote practical applications, it is of great importance to examine the failure mechanism (including joint load-bearing capacity and interface damage level) of the joint under external loading.

Plenty of investigations [1-6] had illustrated that failure takes place progressively as energy dissipates gradually at the crack tip. The progressive failure process is mainly attributed to the mechanical properties of the adhesive and the stress states of the adhesive layer controlled by the geometrical configurations and constraint effects [1-5]. In addition, owing to lower stiffness of the

\footnotetext{
* Corresponding author. Tel.: +8610 8254 4291; fax: +86 1082544256 .

E-mail address: liaohuanxin@hotmail.com (L. Liao).
}

adhesive layer compared with that of the adherend, failure generally occurs in the adhesive layer $[7,8]$.

For the cohesive failure, cohesive zone model (CZM) is widely used to capture the damage onset and growth with mesh independence and dispensable initial crack, maintaining the possibility to characterize the behavior of the structure up to failure [1-8]. In addition, Castagnetti et al. [9] represented the advantages of numerical precision and computational speed by adopting an efficient finite element computational method with the core of CZM. Moreover, Campilho et al. [10] examined the influences of cohesive parameters. As for the mixed-mode strength, Spaggiari et al. [11] stated that the responses in mode I (normal stresses) and mode II (shear stresses) of the adhesive are significantly different. However, the main control parameters that determine the mechanical properties of the adhesive joints have not yet been analyzed systematically. Liao et al. [12] discussed the effects of influential parameters on the load-bearing capacity of single scarf adhesive joint using dimensional analysis preliminarily. Pardoen et al. [13] also investigated the related factors, such as the material properties and the geometry, on the responses during the wedge opening process via dimensional analysis. The previous investigations demonstrated that the influential parameters affect the 
mechanical properties of the adhesive joint collectively rather than individually. However, quantitative analysis with exact expression has not yet been carried out.

In the present study, a double scarf adhesive joint (DSJ) under uniaxial tensile loading was adopted as the research object, in which the interface experiences tensile/shear stresses under external loading. Assuming that the cohesive failure occurs in the adhesive layer, the mechanical performances of DSJ with different adhesive types and various geometries (adhesive thicknesses \& scarf angles) under uniaxial tensile loading were examined by using a mixed-mode CZM with a bilinear shape coupled with a finite element subroutine (performed in ABAQUS ${ }^{18}[14]$ ). Accordingly, the effects of adhesive type and geometry on the mixed-mode failure of adhesive joint were analyzed numerically. The intrinsic parameters of the adhesive with the label Hysol ${ }^{\mathbb{B}}$ EA9361 [15] were chosen as the benchmark values for the cohesive parameters. In the evaluation of the adhesive type, the influences of varied parameters corresponding to the benchmark cohesive values were studied numerically. In addition, the effects of geometry including the adhesive thickness which influences the cohesive parameters [3], and the scarf angle which affects the interface stress state, were also analyzed to identify the failure mechanism of DSJ. Furthermore, mathematical expressions for the ultimate tensile loading and the necessary energy for failure of DSJ with respect to the thickness-dependency cohesive parameters in two modes (I and II) and the scarf angle were examined.

\section{Numerical analysis}

\subsection{DSJ model}

In order to examine the effects of control parameters on the failure mechanism of the adhesive joint, the mechanical performances of DSJ subjected to uniaxial tensile loading, which experiences the tensile/shear stresses state at the adhesive interface, was investigated. Fig. 1 shows a DSJ model, in which two adherends with the same material are bonded together by using the adhesive layer with thickness of $t_{2}$ and scarf angle of $\theta$. Young's modulus and Poisson's ratio of the adherends are denoted as $E_{1}$ (209 GPa) and $\nu_{1}(0.29)$, and those of the adhesive layer are $E_{2}$ and $\nu_{2}$, respectively. The length and the width of DSJ are $2 l_{1}(100 \mathrm{~mm})$ and $2 w(20 \mathrm{~mm})$, respectively.

As a 2D plane-strain problem (thin plate specimen), Cartesian coordinates $(x, y)$ were adopted in modeling. As shown in Fig. 1, with full constraints at the left end of DSJ, the uniaxial tensile loading was simulated by controlling the displacement increment along the $x$-direction $(u)$ at the right end of DSJ.

\subsection{Finite element method}

In the finite element analysis, the geometrical thickness of the adhesive layer (for easier visual effect) is different from the real thickness $t_{2}$. Accordingly, the adhesive layer was built as a single layer using four-node cohesive elements, which share nodes with the neighboring elements in the adherends. The adherends, which

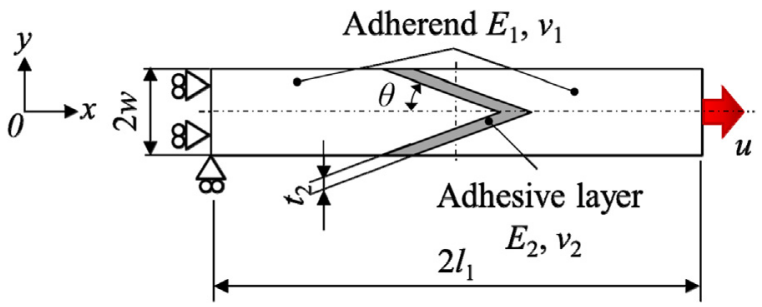

Fig. 1. A DSJ model with boundary conditions. were defined as isotropic elastic for simplicity, were meshed using four-node quadrilateral plane-strain elements. The adhesive region was meshed densely using the biasing effects while the other regions were meshed sparsely for higher computational accuracy and efficiency. In addition, optional viscous damping was implemented between node pairs to improve convergence.

In order to capture the progressive nonlinear failure occurred at the adhesive interface, a material and geometrical nonlinear numerical analysis was performed in ABAQUS ${ }^{\mathbb{R}}$ by adopting a CZM to simulate damage initiation and growth. Bilinear tractionseparation (T-S) curve was adopted as the constitute law for the adhesive layer with definite thickness. In the T-S curve, the cohesive strength $\sigma$ and the critical fracture energy $G$ are the main parameters, which govern the interface separation behavior $[8,16]$. In addition, since the scarf interface is not perpendicular or parallel to the tensile loading, the mixed-mode (mode I and mode II) failure should be taken into account.

Under the mixed-mode condition, damage initiation is controlled by a quadratic stress criterion through the following relation $[2,3,6,10]$,

$\left(\sigma_{I} / \sigma_{u, \mathrm{I}}\right)^{2}+\left(\sigma_{I I} / \sigma_{u, \mathrm{II}}\right)^{2}=1$

where $\sigma_{\mathrm{I}}$ and $\sigma_{\mathrm{II}}$ are the stresses at the interface element in mode I and mode II, while $\sigma_{u, \mathrm{I}}$ and $\sigma_{u, \mathrm{II}}$ are the cohesive strengths of the given adhesive in mode I and mode II, respectively. Correspondingly, the damage level $D$, which can be expressed according to the total displacement jump $\Delta=\sqrt{\left(\delta_{\mathrm{I}}\right)^{2}+\left(\delta_{\mathrm{II}}\right)^{2}}$ [3-5], is given as

$D=\frac{\Delta_{f}\left(\Delta_{\max }-\Delta_{o}\right)}{\Delta_{\max }\left(\Delta_{f}-\Delta_{o}\right)}$

where $\Delta_{o}$ and $\Delta_{f}$ are the total displacements for damage onset and complete failure, respectively; $\Delta_{\max }$ represents the maximum total displacement ever experienced during the loading history; $\Delta_{f}$ is calculated by $\Delta_{f}=2 G / \sqrt{\left(\delta_{o, \mathrm{I}}\right)^{2}+\left(\delta_{o, \mathrm{II}}\right)^{2}}$, where $G$ is the total energy released during the separation of the adhesive layer.

In addition, a linear fracture criterion is chosen to determine the damage propagation, which is expressed as $[2,3,6,10]$

$G_{\mathrm{I}} / G_{\mathrm{Ic}}+G_{\mathrm{II}} / G_{\mathrm{II}}=1$

\section{Results and discussions}

The load-bearing capacity of the adhesive joints has been extensively estimated by employing the ultimate loading [17-20], which is denoted as $F_{u}$. In addition, the failure energy $E_{f}$ was also introduced to evaluate the joint performance [12] as

$E_{f}=\int_{0}^{u_{u}} F d u$

where $u_{u}$ is the ultimate displacement corresponding to the resultant loading $F$ dropping from the ultimate value to zero, which indicates complete failure. The physical significance of the failure energy is to illustrate the energy required for the failure of the joint with a given scarf angle and selected adhesive. In this study, the effects of the adhesive type and the geometry of the adhesive layer were examined, respectively.

\subsection{Effect of adhesive type}

As for the effect of the adhesive type, the real dominant factor is the cohesive parameters. The benchmark values were chosen as the cohesive parameters of the selected adhesive (a ductile adhesive with the label Hysol ${ }^{\mathbb{B}}$ EA9361 [15]). However, Xu and Wei [3] pointed out that the cohesive parameters are adhesive thicknessdependency. To eliminate the influence of the adhesive thickness on 
the properties of the adhesive, the thickness of the adhesive layer was fixed as $0.5 \mathrm{~mm}$. In addition, the scarf angle $\theta$ was chosen as $30^{\circ}$. Subsequently, the cohesive strength $\sigma_{u, i}(i=\mathrm{I}, \mathrm{II})$ and the critical fracture energy $G_{i c}(i=\mathrm{I}, \mathrm{II})$ in mode I and mode II are the main control parameters to influence the mechanical properties of DSJ under uniaxial tensile loading. According to our previous investigation [12], the non-dimensional-normalized form for the properties of the adhesive is $\left(G_{\mathrm{Ic}} / \sigma_{u, \mathrm{I}} t_{2}, G_{\mathrm{II}} / G_{\mathrm{Ic}}, \sigma_{u, \mathrm{II}} / \sigma_{u, \mathrm{I}}\right)$, in which the latter two indicate the extent of the anisotropy of the adopted adhesive between mode I and mode II.

At first, the extent of the anisotropy of the adopted adhesive between mode I and mode II was held as constant, which was denoted as Case 1 . The ratios $(r)$ of the assigned cohesive strength in mode I to the intrinsic cohesive strength in mode I (13.57 MPa) were 0.25 (3.39 MPa), 0.5 (6.78 MPa), 1.0 (13.57 MPa), 1.5 (20.36 MPa), and $2.0(27.14 \mathrm{MPa})$, respectively. In addition, the assigned cohesive strength in mode II, the critical fracture energy in mode I and mode II were set according to the equal ratios mentioned above. Consequently, all the assigned parameters of the constitutive relation of the adhesive are determined as shown in Table 1.

Fig. 2 shows the variation of the non-dimensional-normalized form of the ultimate tensile loading $F_{u} / \sigma_{u, 1} t_{2}$ with respect to the ratio $r$, in which the purple solid line is the average value of the five scatter points. It can be found that the ultimate tensile loading $F_{u}$ is influenced only by the cohesive strength in mode I $\sigma_{u, \mathrm{I}}$ when the extent of the anisotropy of the adopted adhesive between mode I and mode II is held as constant. In addition, there is a linear relationship between $F_{u}$ and $\sigma_{u, \mathrm{I}}(r)$. Furthermore, it should be noted that the results prove the rationality of the nondimensional-normalized form of the ultimate tensile loading [12] $\left(F_{u} / \sigma_{u, \mathrm{I}} t_{2}=f\left(G_{\mathrm{I} c} / \sigma_{u, \mathrm{I}} t_{2}, G_{\mathrm{II} c} / G_{\mathrm{I} c}, \sigma_{u, \mathrm{II}} / \sigma_{u, \mathrm{I}}\right)\right)$ when takes the effect of the adhesive type into account only.

Hence, in the next, the effect of $\sigma_{u, \mathrm{I}}$ on the performance of DSJ was also examined, denoted as Case 2 . The ratios $r_{\sigma}^{\mathrm{I}}$ between the assigned $\sigma_{u, \mathrm{I}}$ and the intrinsic value of $\mathrm{Hysol}^{\mathrm{E}}$ EA9361 were chosen as 0.25 (3.39 MPa), 0.5 (6.78 MPa), 1.0 (13.57 MPa), 1.5 (20.36 MPa), and $2.0(27.14 \mathrm{MPa})$, respectively. On the other hand, the assigned cohesive strength in mode II and the critical fracture energy in two modes (I and II) were maintained as constant values, which are equal to the intrinsic values of the given adhesive. The corresponding cohesive parameters are listed in Table 2.

Finally, the effect of critical fracture energy on the mechanical properties of DSJ was evaluated, denoted as Case 3, in which the cohesive strength in each mode was maintained constantly. The critical fracture energy in mode II $\left(G_{\text {IIC }}\right)$ was set as a constant, which equals to the intrinsic value of the selected adhesive $(3.96 \mathrm{~N} / \mathrm{mm})$. In addition, the ratios $r_{G}^{\mathrm{I}}$ of the assigned $G_{\text {Ic }}$ to the intrinsic value of Hysol $^{\text {is }}$ EA9361 were selected as $0.25(0.99 \mathrm{~N} /$ $\mathrm{mm}), 0.5(1.98 \mathrm{~N} / \mathrm{mm}), 1.0(3.96 \mathrm{~N} / \mathrm{mm}), 1.5(5.95 \mathrm{~N} / \mathrm{mm})$ and 2.0 $(7.92 \mathrm{~N} / \mathrm{mm})$, respectively. The corresponding cohesive parameters are listed in Table 3.

Table 1

Case 1 - Assigned cohesive parameters of adhesive (constant anisotropy extent $t_{2}=0.5 \mathrm{~mm}$ )

\begin{tabular}{|c|c|c|c|c|c|}
\hline \multirow[t]{2}{*}{ Parameters } & \multicolumn{5}{|c|}{$r\left(\sigma_{u, \mathrm{I}} / \sigma_{u, \mathrm{II}}=\right.$ const., $G_{\mathrm{Ic}} / G_{\mathrm{IIC}}=$ const. $)$} \\
\hline & 0.25 & 0.5 & 1.0 (intrinsic) & 1.5 & 2.0 \\
\hline $\begin{array}{l}\text { Cohesive strength (mode I) } \sigma_{u, \mathrm{I}} \\
\quad(\mathrm{MPa})\end{array}$ & 3.39 & 6.78 & 13.57 & 20.36 & 27.14 \\
\hline $\begin{array}{l}\text { Cohesive strength (mode I) } \sigma_{u, \mathrm{II}} \\
\quad(\mathrm{MPa})\end{array}$ & 4.37 & 8.74 & 17.48 & 26.23 & 34.96 \\
\hline $\begin{array}{l}\text { Critical fracture energy (mode I) } G_{\text {Ic }} \\
(\mathrm{N} / \mathrm{mm})\end{array}$ & 0.99 & 1.98 & 3.96 & 5.95 & 7.92 \\
\hline $\begin{array}{l}\text { Critical fracture energy (mode II) } \\
\text { G IIc }_{(\mathrm{N} / \mathrm{mm})}\end{array}$ & 1.64 & 3.28 & 6.57 & 9.86 & 13.14 \\
\hline
\end{tabular}

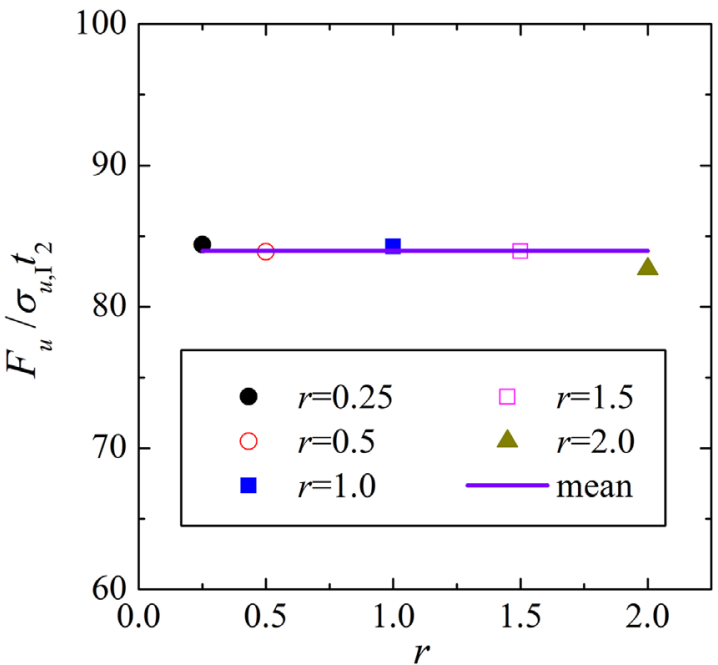

Fig. 2. Non-dimensional-normalized form $\left(F_{u} / \sigma_{u, \mathrm{I}} t_{2}\right)$-ratio $(r)$ curves of DSJ under uniaxial tensile loading $\left(t_{2}=0.5 \mathrm{~mm}, \theta=30^{\circ}, \sigma_{u, \mathrm{I}} / \sigma_{u, \mathrm{II}}=\right.$ const., $G_{\mathrm{Ic}} / G_{\mathrm{IIC}}=$ const. $)$.

\section{Table 2}

Case 2 - Assigned cohesive parameters of adhesive (constant critical fracture energy in mode I and mode II, $t_{2}=0.5 \mathrm{~mm}$ ).

\begin{tabular}{|c|c|c|c|c|c|}
\hline \multirow[t]{2}{*}{ Parameters } & \multicolumn{5}{|c|}{$r_{\sigma}^{\mathrm{I}}\left(\sigma_{u, \mathrm{II}}=\right.$ const., $G_{\mathrm{Ic}} / G_{\mathrm{IIC}}=$ const. $)$} \\
\hline & 0.25 & 0.5 & 1.0 (intrinsic) & 1.5 & 2.0 \\
\hline $\begin{array}{l}\text { Cohesive strength (mode I) } \sigma_{u, \mathrm{I}} \\
(\mathrm{MPa})\end{array}$ & 3.39 & 6.78 & 13.57 & 20.36 & 27.14 \\
\hline $\begin{array}{l}\text { Cohesive strength (mode I) } \sigma_{u, \mathrm{II}} \\
(\mathrm{MPa})\end{array}$ & 17.48 & 17.48 & 17.48 & 17.48 & 17.48 \\
\hline $\begin{array}{l}\text { Critical fracture energy (mode I) } \\
G_{\text {Ic }}(\mathrm{N} / \mathrm{mm})\end{array}$ & 3.96 & 3.96 & 3.96 & 3.96 & 3.96 \\
\hline $\begin{array}{l}\text { Critical fracture energy (mode II) } \\
G_{\text {IIc }}(\mathrm{N} / \mathrm{mm})\end{array}$ & 6.57 & 6.57 & 6.57 & 6.57 & 6.57 \\
\hline
\end{tabular}

Table 3

Case 3 - Assigned cohesive parameters of adhesive (constant cohesive strength in mode I and mode II, $t_{2}=0.5 \mathrm{~mm}$ ).

\begin{tabular}{|c|c|c|c|c|c|}
\hline \multirow[t]{2}{*}{ Parameters } & \multicolumn{5}{|c|}{$r_{G}^{\mathrm{I}}\left(\sigma_{u, \mathrm{I}} / \sigma_{u, \mathrm{II}}=\right.$ const., $G_{\mathrm{IIC}}=$ const. $)$} \\
\hline & 0.25 & 0.5 & 1.0 (intrinsic) & 1.5 & 2.0 \\
\hline $\begin{array}{l}\text { Cohesive strength (mode I) } \sigma_{u, \mathrm{I}} \\
\quad(\mathrm{MPa})\end{array}$ & 13.57 & 13.57 & 13.57 & 13.57 & 13.57 \\
\hline $\begin{array}{l}\text { Cohesive strength (mode I) } \sigma_{u, \mathrm{II}} \\
\quad(\mathrm{MPa})\end{array}$ & 17.48 & 17.48 & 17.48 & 17.48 & 17.48 \\
\hline $\begin{array}{l}\text { Critical fracture energy (mode I) } \\
G_{\text {Ic }}(\mathrm{N} / \mathrm{mm})\end{array}$ & 0.99 & 1.98 & 3.96 & 5.95 & 7.92 \\
\hline $\begin{array}{l}\text { Critical fracture energy (mode II) } \\
G_{\text {IIc }}(\mathrm{N} / \mathrm{mm})\end{array}$ & 6.57 & 6.57 & 6.57 & 6.57 & 6.57 \\
\hline
\end{tabular}

Accordingly, the effects of the three cases on the mechanical properties of DSJ under uniaxial tensile loading were examined through evaluating the influences on the ultimate tensile loading $F_{u}$, the failure energy $E_{f}$ and the interface damage level $D$, respectively.

\subsubsection{Influence on ultimate tensile loading $F_{u}$}

Fig. 3 shows the variation of the ultimate tensile loading $F_{u}$ with the ratios of $r$ (Case 1), $r_{\sigma}^{\mathrm{I}}$ (Case 2) and $r_{G}^{\mathrm{I}}$ (Case 3), respectively. In Case 1, the ultimate tensile loading $F_{u}$ linearly increases with $r$. Therefore the form of fitting curve can be chosen as the power function of $F_{u}=a r^{b}$, where the power $b$ is 1 .

In Case 2, the ultimate tensile loading $F_{u}$ increases with $r_{\sigma}^{\mathrm{I}}$. According to the non-dimensional-normalized form of the ultimate tensile loading $F_{u} / \sigma_{u, \mathrm{I}} t_{2}=f\left(G_{\mathrm{Ic}} / \sigma_{u, \mathrm{I}} t_{2}, G_{\mathrm{II} c} / G_{\mathrm{II}}, \sigma_{u, \mathrm{II}} / \sigma_{u, \mathrm{I}}\right)$, the cohesive 

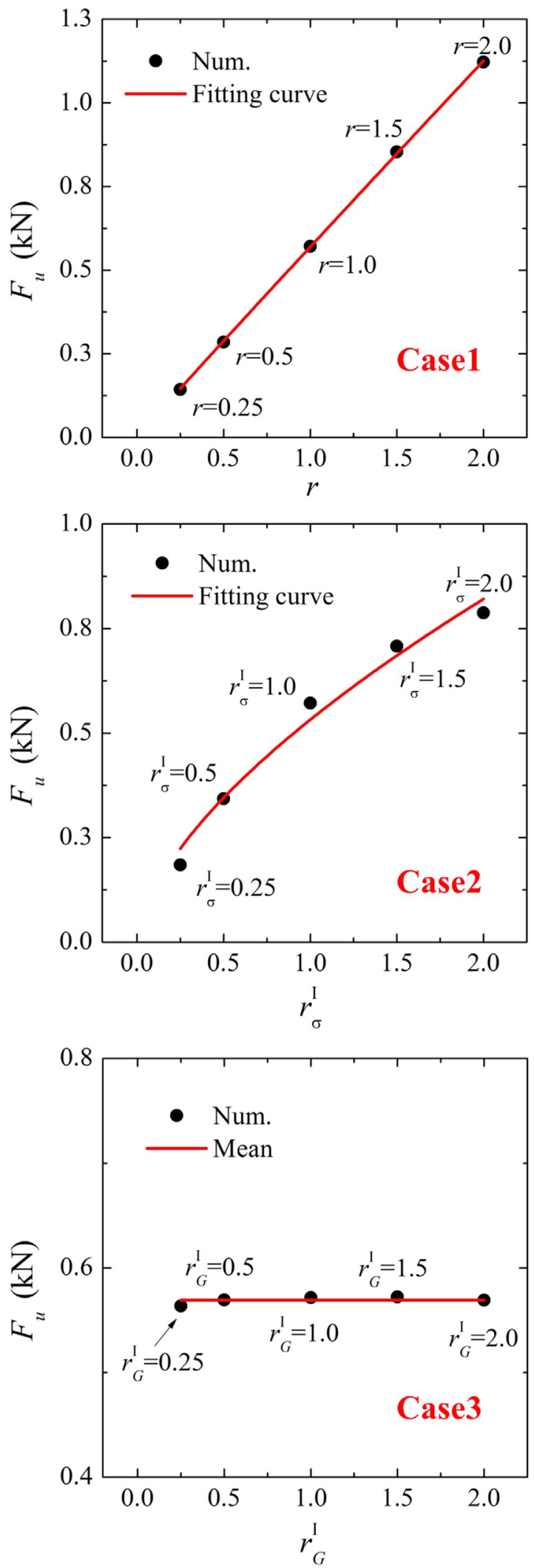

Fig. 3. Variation of the ultimate tensile loading $F_{u}$ with the ratios of $r, r_{\sigma}^{\mathrm{I}}$ and $r_{G}^{\mathrm{I}}$, respectively $\left(t_{2}=0.5 \mathrm{~mm}, \theta=30^{\circ}\right.$, Case $1: \sigma_{u, \mathrm{I}} / \sigma_{u, \mathrm{II}}=$ const., $G_{\mathrm{IC}} / G_{\mathrm{IIC}}=$ const., Case $2: \sigma_{u}$ II $=$ const., $G_{\text {Ic }} / G_{\text {IIC }}=$ const., Case 3: $\sigma_{u, I} / \sigma_{u, I I}=$ const., $G_{\text {IIC }}=$ const. ).

strength in mode I $\left(\sigma_{u, \mathrm{I}}\right)$ is the control parameter to influence $F_{u}$. Consequently, the relationship between them $\left(F_{u}\right.$ and $\left.\sigma_{u, 1}\right)$ is revealed by adopting the power function $F_{u}=a\left(r_{\sigma}^{\mathrm{I}}\right)^{b}$ with the power $b=0.6$,
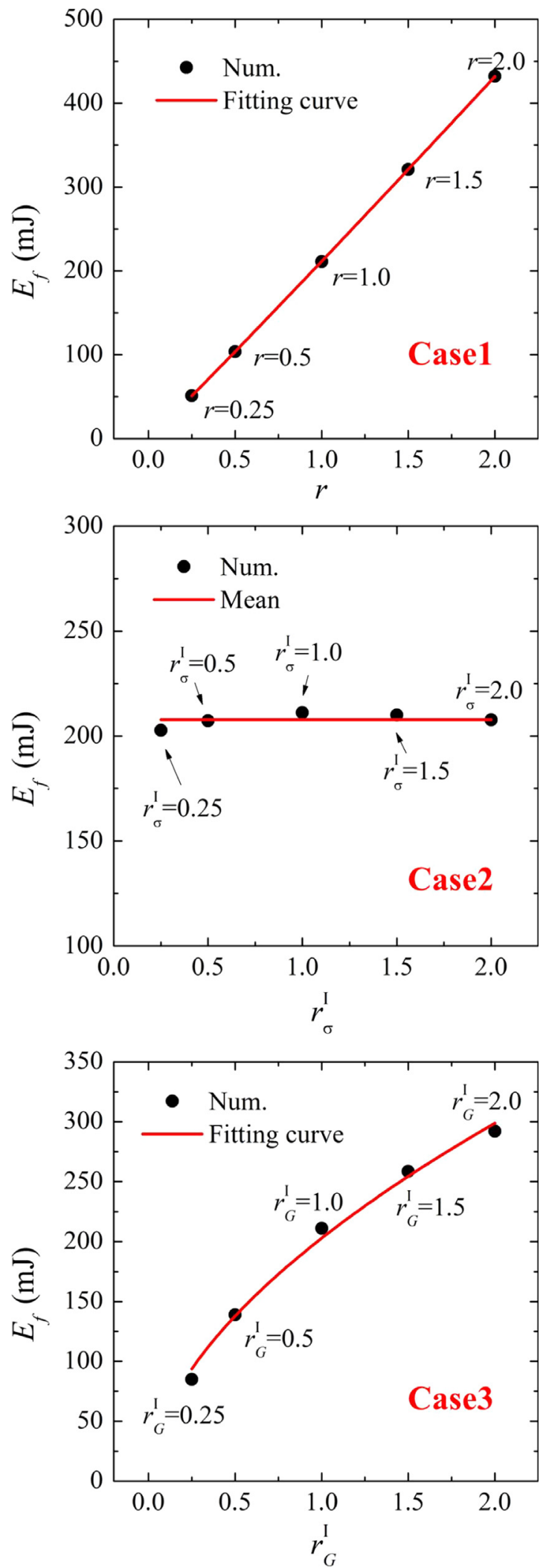

Fig. 4. Variation of the failure energy $E_{f}$ with the ratios $r, r_{\sigma}^{\mathrm{I}}$ and $r_{G}^{\mathrm{I}}$, respectively $\left(t_{2}=0.5 \mathrm{~mm}, \theta=30^{\circ}\right.$, Case $1: \sigma_{u, \mathrm{I}} / \sigma_{u, \mathrm{II}}=$ const., $G_{\mathrm{Ic}} / G_{\mathrm{IIC}}=$ const., Case $2: \sigma_{u, \mathrm{II}}=$ const., $G_{\text {Ic }} / G_{\text {IIc }}=$ const., Case 3: $\sigma_{u, I} / \sigma_{u, \mathrm{II}}=$ const., $G_{\text {IIc }}=$ const. ).

which demonstrates that the variation of $F_{u}$ is nonlinear with $\sigma_{u, \mathrm{I}}$. Combined with the results obtained in Case 1, it can be concluded that the both cohesive strengths in mode I and mode II contribute to the variation of the ultimate tensile loading $F_{u}$. 
In Case 3, the variation of the critical fracture energy has no influence on the ultimate tensile loading $F_{u}$, which indicates that the ultimate tensile loading $F_{u}$ is mainly determined by the cohesive strength.

\subsubsection{Influence on failure energy $E_{f}$}

Taking the ultimate tensile loading and the ultimate applied displacement into account, the variation of failure energy $E_{f}$ with respect to the ratios of $r$ (Case 1 ), $r_{\sigma}^{\mathrm{I}}$ (Case 2) and $r_{G}^{\mathrm{I}}$ (Case 3) was examined, respectively. As shown in Fig. 4, in Case 1, the failure energy $E_{f}$ varies with the ratio $r$ in direct proportion. Assuming the fitting curve form for the scatter points as a power function $E_{f}=a r^{b}$ (where the power $b$ is 1 ), it can be observed that the relationship between the failure energy $E_{f}$ of DSJ and the ratio $r$ is linear.

As for the influences of $r_{\sigma}^{\mathrm{I}}$ on the failure energy $E_{f}$ (Case 2), the value of $E_{f}$ shows slight variation with various $r_{\sigma}^{\mathrm{I}}$, which means the effect of the ratio $r_{\sigma}^{\mathrm{I}}$ on $E_{f}$ can be ignored. On the other hand, the relationship between the failure energy $E_{f}$ of DSJ and the ratio $r_{G}^{\mathrm{I}}$ is described in Fig. 4 of the Case 3. With increasing the ratio $r_{G}^{\mathrm{I}}$, the failure energy $E_{f}$ increases nonlinearly. The $E_{f}$ function with respect to the ratio $r_{G}^{\mathrm{I}}$ is fitted using the power function $E_{f}=a\left(r_{G}^{\mathrm{I}}\right)^{b}$, where the power $b$ is fitted as 0.6 .

According to the results obtained in Case 1 to Case 3, the failure energy $E_{f}$ of DSJ is governed by the critical fracture energy in mode I and mode II together. In addition, it also can be obtained that the cohesive strength in each mode has no influence on the failure energy $E_{f}$ of DSJ.

\subsubsection{Influence on interface damage level $D$}

Corresponding to the ultimate tensile loading $F_{u}$, the damage level $D$ of the adhesive layer for the three cases were also investigated. Fig. 5 shows the damage distributions at the interface of DSJ corresponding to the ultimate tensile loading. In Case 1, it can be observed that the damage level $D$ decreases as the ratio $r$ increases. In addition, during the process of decreasing the ratio $r$, the distribution of damage level at the interface tends to be uniform.

In Case 2, as an increase of the ratio $r_{\sigma}^{\mathrm{I}}$, the damage level $D$ also decreases. However, different from the results of Case 1, it is seen that the distribution of the interface damage level becomes more uniform as the ratio $r_{\sigma}^{\mathrm{I}}$ increases.

In Case 3, it is seen that the interface damage level $D$ is almost invariable for the ratio $r_{G}^{\mathrm{I}}$ between 1.0 and 1.5 except when the ratio $r_{G}^{\mathrm{I}}$ is 2.0. For the cases discussed in the present study, the critical fracture energies in mode $\mathrm{I}\left(G_{\mathrm{IC}}\right)$ are smaller than those in mode II $\left(G_{\text {IIC }}\right)$. However, when the ratio $r_{G}^{\mathrm{I}}$ is 2.0 , the situation is different with the larger critical fracture energy in mode I $\left(G_{I C}\right)$ compared to that in mode II $\left(G_{\text {IIC }}\right)$.

In summary, the ultimate tensile loading of DSJ is mainly determined by the cohesive strengths in mode I and mode II together; the failure energy of DSJ is governed by the critical fracture energies in mode I and mode II cooperatively; the damage level is also significantly influenced by the cohesive parameters. The detailed effect contributed by the cohesive parameters in each mode on the mechanical properties of DSJ will be examined in Section3.2 to explain the failure mechanism of the joint.

\subsection{Effect of geometry}

Adhesive thickness is another important factor in determining the joint mechanical properties, whose effect is combined with all cases as other variables are involved, such as the type of loading (shear, peel, or cleavage), the adherend behavior (elastic or plastic), and the type of adhesive (ductile or brittle) [2]. In the present study, the type of loading is tension. However, the scarf angle and
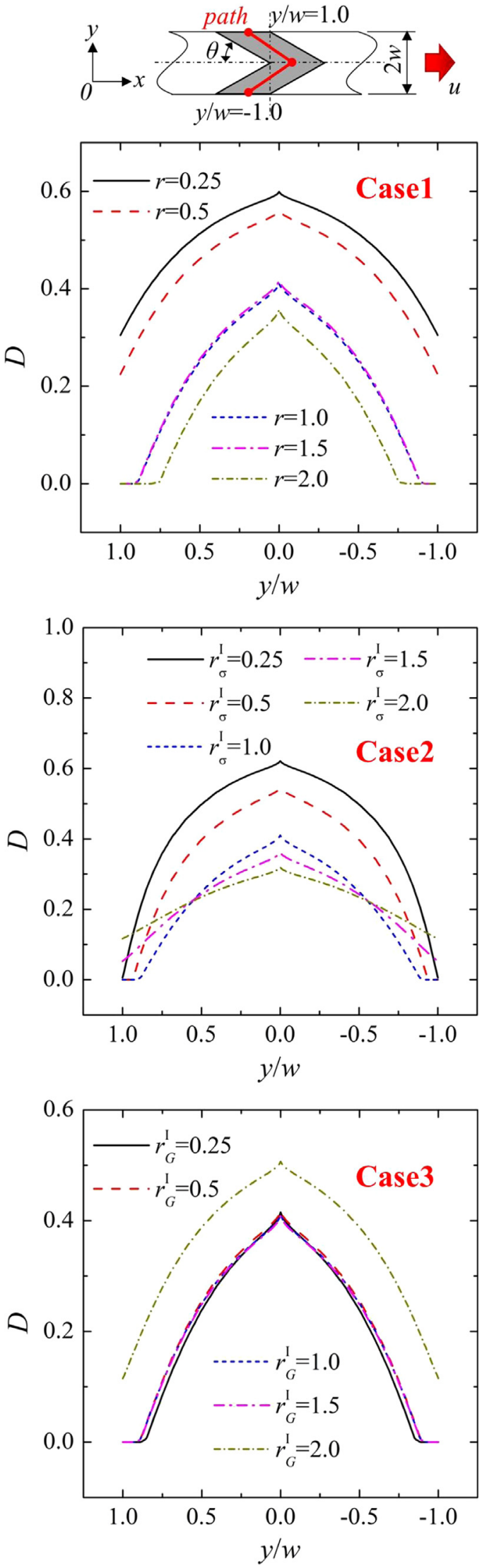

Fig. 5. Damage distributions at the interface of DSJ corresponding to the ultimate tensile loading with respect to the ratios $r, r_{\sigma}^{\mathrm{I}}$ and $r_{G}^{\mathrm{I}}$, respectively $\left(t_{2}=0.5 \mathrm{~mm}\right.$, $\theta=30^{\circ}$, Case 1: $\sigma_{u, \mathrm{I}} / \sigma_{u, \mathrm{II}}=$ const., $G_{\mathrm{II}} / G_{\mathrm{IIC}}=$ const., Case $2: \sigma_{u, \mathrm{II}}=$ const., $G_{\mathrm{Ic}} / G_{\text {IIc }}=$ const., Case 3: $\sigma_{u, \mathrm{I}} / \sigma_{u, \mathrm{II}}=$ const., $\mathrm{G}_{\mathrm{IIC}}=$ const. ). 


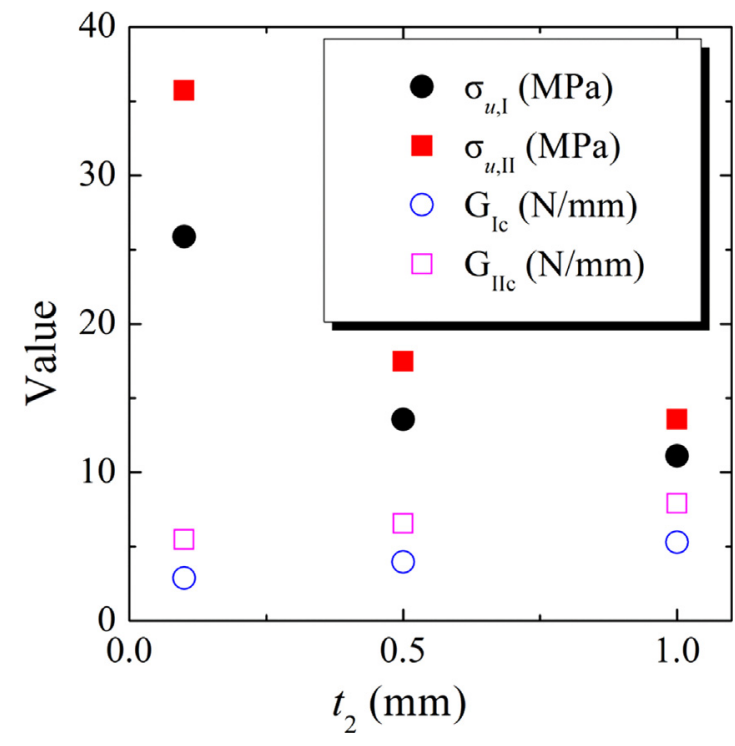

Fig. 6. Adhesive thickness-dependency cohesive parameters [12].

the uniaxial tensile loading affect the stress state at the interface together. To simplify the analysis, the adherend behavior and the skeleton dimensions (the length $2 l_{1}$ and the width $2 w$ of DSJ) are remained unchanged. In addition, the type of adhesive is also selected as Hysol $^{\mathbb{E}}$ EA9361, which is a ductile adhesive [15]. Consequently, the adhesive thickness $t_{2}$ and the scarf angle $\theta$ become the geometrical factors that influence the joint performance.

The adhesive thickness was chosen as $0.1,0.5$ and $1.0 \mathrm{~mm}$ to evaluate the effect on the joint mechanical property. In addition, the scarf angle $\theta$ was set as $0^{\circ}$ (single-lap joint), $15^{\circ}, 30^{\circ}, 45^{\circ}, 60^{\circ}$, $75^{\circ}$ and $90^{\circ}$ (butt joint), respectively. The adhesive thicknessdependency cohesive parameters are showed as in Fig. 6 [12].

Fig. 7 indicates that the ultimate tensile loading $F_{u}$ of DSJ decreases as the scarf angle $\theta$ increases for each given adhesive thickness $t_{2}$. In the case of the joint with the scarf angle $\theta=0^{\circ}$, the stress state at the interface is pure shear stress. Hence the ultimate tensile loading $F_{u}$ of the joint is determined only by the cohesive strength in mode II $\left(\sigma_{u, \mathrm{II}}\right)$ of the selected adhesive. However, for the joint with the scarf angle $\theta=90^{\circ}$, where pure tensile stress occurs at the interface, the ultimate tensile loading $F_{u}$ of DSJ is just governed by the cohesive strength in mode I $\left(\sigma_{u, \mathrm{I}}\right)$ of the adhesive. Davies et al. [21] reported that a decrease of tension/shear loads as the adhesive thickness increases, which coincides with the results obtained in the present study (as shown in Fig. 7). In addition, as the thickness increases, a significant drop in strength and failure strain under pure tension, and a smaller reduction under tension/shear and pure shear loads have been reported [21]. According to their conclusions [21], it can be assumed that the cohesive strength in mode I $\left(\sigma_{u, \mathrm{I}}\right)$ and mode II $\left(\sigma_{u, \mathrm{II}}\right)$ make different contributions to the ultimate tensile loading $F_{u}$ of DSJ. In the present study, it is assumed that two coefficients $\alpha$ and $\beta$ represent the corresponding contribution in each mode to the ultimate tensile loading $F_{u}$ of DSJ. Subsequently, $\alpha$ and $\beta$ were calculated using the ratio of the ultimate tensile/shear loading to the cohesive strength in each mode, respectively. For the joint with scarf angle $0^{\circ}<\theta<90^{\circ}$, except for the intrinsic effect of each cohesive strength in each mode, derivational coefficients resulting from the variation of stress state at the interface should also be taken into account. At the scarf interface, the direction of decomposition of the component in each mode should be the uniaxial, which is the same as the tensile loading applied on the DSJ. As far as the cohesive strength in each mode and the interface stress state are concerned, the expression of the ultimate tensile loading $F_{u}$ is the function of $f$ $\left(\sigma_{u, \mathrm{I}}, \sigma_{u, \mathrm{II}}, \theta\right)$, in which the coefficients of the component in each

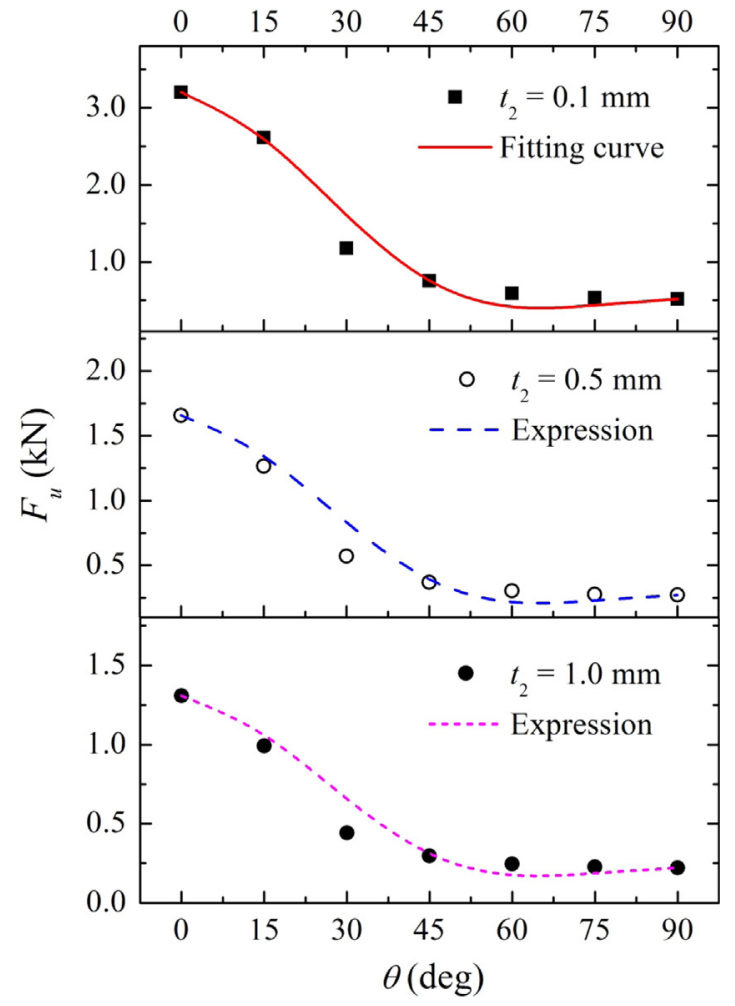

Fig. 7. Variation of the ultimate tensile loading $F_{u}$ with the scarf angle $\theta$ at each given adhesive thickness $t_{2}$

mode is denoted as $c_{1}$ and $c_{2}$, respectively. Accordingly, the function form can be given as

$$
\begin{aligned}
F_{u}= & c_{1} \sigma_{u, \mathrm{I}} \sin \theta+c_{2} \sigma_{u, \mathrm{II}} \cos \theta=\left(\alpha \sin ^{n} \theta\right) \sigma_{u, \mathrm{I}} \sin \theta \\
& +\left(\beta \cos ^{n} \theta\right) \sigma_{u, \mathrm{II}} \cos \theta
\end{aligned}
$$

where $n$ was determined as 4 by fitting the scatter points in Fig. 7 in the case of the adhesive thickness $t_{2}=0.1 \mathrm{~mm}$ (upper picture). As shown in Fig. 7, the results obtained by the expressions mentioned above show good agreement with the scatter points for different adhesive thickness $t_{2}$, which indicates the availability and rationality of the obtained expressions.

Fig. 8 shows the variation of the failure energy $E_{f}$ of DSJ with the scarf angle in each adhesive thickness $t_{2}$. By adopting CZM as the constitutive relation of the adhesive layer, the failure energy of DSJ is governed by the critical fracture energies in mode I and mode II together with the tensile/shear stress state at the interface. Referred to the analysis method for the ultimate tensile loading shown in Fig. 7, the expression can also be assumed as

$E_{f}=c_{3} G_{I C} \sin \theta+c_{4} G_{\text {IIC }} \cos \theta=\left(\gamma \sin ^{n} \theta\right) G_{I C} \sin \theta+\left(\varphi \cos ^{n} \theta\right) G_{I I C} \cos \theta$

where $\gamma$ and $\varphi$ are two coefficients indicating the contribution in each mode to failure energy $E_{f}$ of DSJ, which are the ratios of the failure energy in pure tensile/shear to the critical fracture energy in each mode, respectively. Subsequently, $c_{3}$ and $c_{4}$ are the coefficients representing the component for failure energy in each mode. In addition, the Power $n$ was also chosen as 4 , which equals to the value in Eq. (5). By comparing the expressions with the scatter points in Fig. 8, it can be obtained that Eq. (6) accurately reveals the relationship between the failure energy of DSJ and the interface stress state. Furthermore, with the same form of expressions for ultimate tensile loading $F_{u}$ and failure energy $E_{f}$ of DSJ, especially with the same value of the power in expressions, Eqs. (5) and (6) present that the contributions of the cohesive parameters in mode I and mode II to the mixed-mode failure of 


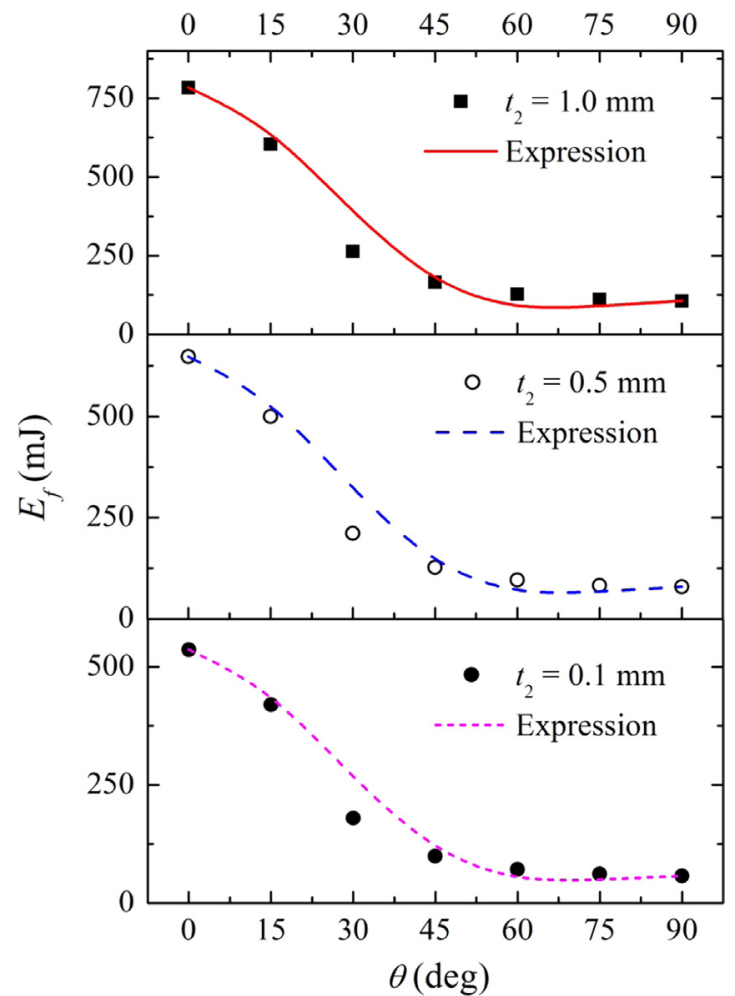

Fig. 8. Variation of the failure energy $E_{f}$ with the scarf angle $\theta$ at each given adhesive thickness $t_{2}$.
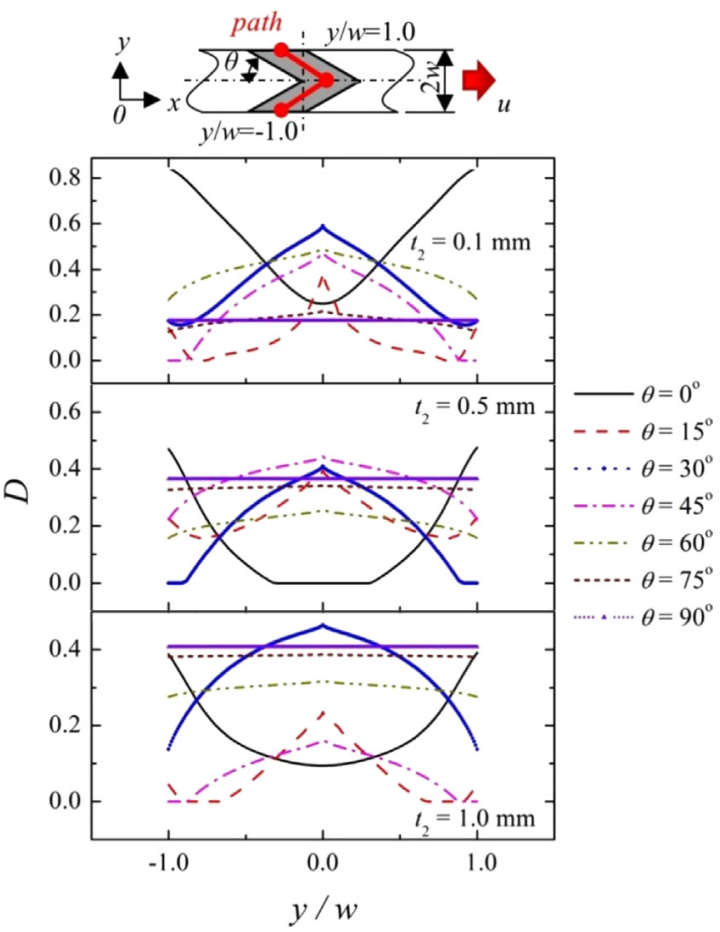

Fig. 9. Damage distributions at the interface of DSJ corresponding to the ultimate loading with respect to the adhesive thickness $t_{2}$ at given scarf angle $\theta$.

DSJ (with tensile/shear stress state at the interface) are different. Returning to the results shown in Fig. 5 (Case 3, $r_{G}^{\mathrm{I}}=2.0$ ), when the critical fracture energy in mode $\mathrm{I}\left(G_{\mathrm{Ic}}\right)$ is larger than that in mode II $\left(G_{\text {IIC }}\right)$, the contributions of two modes to the joint performance are changed. Essentially, owing to the change of the adhesive properties, the mechanical properties of DSJ vary accordingly.
Fig. 9 shows the variation of the damage level $D$ for various adhesive thicknesses $t_{2}$ and scarf angles $\theta$. It can be seen that the variation trend of the damage for specific scarf angle is greatly dependent on the adhesive thickness.

- As shown in Fig. 9, with increasing adhesive thickness $t_{2}$, the damage levels $D$ tend to uniformization. Hence in the case of thinner adhesive thickness, the poor uniformity of loading distribution over the cohesive zone should be considered. However, the relationship between the damage level $D$ of DSJ and the adhesive thickness $t_{2}$ is not monotonous.

- For the joint with scarf angle $\theta=0^{\circ}$ (pure shear, single-lap joint), the damage levels at the two edges $(y / w= \pm 1.0)$ are much higher than those at the middle area of the adhesive layer. The extent of the nonuniformity of the damage level along the interface is higher than the joints with larger scarf angles.

- For the joint with scarf angle $0^{\circ}<\theta<90^{\circ}$, owing to the geometrical configuration of the V-type interface, the stress concentration occurs at the top of the V-type, which leads to high damage level. In addition, the variation of the interface damage level with respect to the scarf angle is not monotonous with each adhesive thickness $t_{2}$. The effects of the scarf angle and the adhesive thickness on the mixed-mode failure are coupled rather than independently.

\section{Conclusions}

In the present study, the effects of the adhesive type and geometry on mixed-mode failure of DSJ under uniaxial tensile loading were examined numerically. With the assumption of the cohesive failure at the interface, the finite element subroutine (performed in ABAQUS) coupled a mixed-mode CZM with a bilinear shape was adopted as the simulated method. According to the nondimensional-normalized form of the ultimate tensile loading in our previous investigation [12], the effect of the adhesive type (cohesive parameters in essence) on the ultimate tensile loading, the failure energy of DSJ and the interface damage level were examined corresponding to three different cases. In addition, the effects of the adhesive thickness and the scarf angle as the geometrical factors on the mechanical properties of DSJ were also analyzed.

(1) The numerical results demonstrate that the cohesive strengths in mode I and mode II control the ultimate tensile loading together but with different contributions. In addition, the critical fracture energies in mode I and mode II, also with different rates, codetermine the failure energy of DSJ.

(2) The mathematical expressions of the ultimate tensile loading and the failure energy of DSJ with respect to the thicknessdependency cohesive parameters in two modes (I and II) and the scarf angle were provided to identify the extents of contribution in each mode component for given type of adhesive.

(3) The relationship between the interface damage level and the adhesive thickness is not monotonous. However, as an increase of adhesive thickness, the uniformity of damage level distributions becomes intensified.

(4) The variation of the interface damage level with the scarf angle is not monotonous for each adhesive thickness. The effects of the scarf angle and the adhesive thickness on the mixed-mode failure are coupled rather than individually. 


\section{Acknowledgements}

This study is supported by the National Natural Science Foundation of China, Grant no. 11202222. The authors would like to gratefully acknowledge.

\section{References}

[1] Adams RD, Wake WC, Comyn J. Structural adhesive joints in engineering. Chapman and Hall; 1997.

[2] da Silva LFM, Rodrigues TNSS, Figueiredo MAV, de Moura MFSF, Chousal JAG. Effect of adhesive type and thickness on the lap shear strength. J Adhes 2006;82:1091-115.

[3] Xu W, Wei Y. Influence of adhesive thickness on local interface fracture and overall strength of metallic adhesive bonding structures. Int J Adhes Adhes 2013;40:158-67.

[4] $\mathrm{Xu} \mathrm{W}$, Wei Y. Assessments for impact of adhesive properties: modeling strength of metallic single lap joint. J Adhes Sci Technol 2013;27:9-29.

[5] Xu W, Wei Y. Strength and interface failure mechanism of adhesive joints. Int J Adhes Adhes 2012;34:80-92.

[6] Campilho RDSG, de Moura MFSF, Ramantani DA, Morais JJL, Domingues JJMS. Tensile behavior of three-dimensional carbon-epoxy adhesively bonded single- and double-strap repairs. Int J Adhes Adhes 2009;29:678-86.

[7] Rudawska Anna. Adhesive joint strength of hybrid assemblies: titanium sheetcomposites and aluminium sheet-composites - experimental and numerical verification. Int J Adhes Adhes 2010;30:574-82.

[8] Peter Gustafson A, Anthony Waas M. The influence of adhesive constitutive parameters in cohesive zone finite element models of adhesively bonded joints. Int J Solids Struct 2009;46:2201-15.
[9] Castagnetti D, Dragoni E, Spaggiari A. Failure analysis of complex bonded structures: experimental tests and efficient finite element modeling by tied mesh method. Int J Adhes Adhes 2011;31:338-46.

[10] Campilho RDSG, Banea MD, Neto JABP, da Silva LFM. Modelling of single-lap joints using cohesive zone models: effect of the cohesive parameters on the output of the simulations. J Adhes 2012;88:513-33.

[11] Spaggiari A, Castagnetti D, Dragoni E. Mixed-mode strength of thin adhesive films: experimental characterization through a tubular specimen with reduced edge effect. J Adhes 2013;89:660-75.

[12] Liao L, Huang C, Sawa T. Effect of adhesive thickness, adhesive type and scarf angle on the mechanical properties of scarf adhesive joints. Int J Solids Struct 2013;50:4333-40.

[13] Pardoen T, Ferracin T, Landis CM, Delannay F. Constraint effects in adhesive joint fracture. Int J Mech Phys Solids 2005;53:1951-83.

[14] Abaqus ${ }^{\mathbb{R}}$ 6.10 Analysis User's Manual, vol. IV: Elements, (C Dassault Systemes 2010.

[15] Hysol ${ }^{\circledR}$ EA9361 Data Sheet, Henkel Corporation, Aerospace Group, 2850 Willow Pass Road, P.O. Box 312, Bay Point, CA 94565 USA, 925.458.8000.

[16] Sørensen BF, Kirkegaard P. Determination of mixed mode cohesive laws. Eng Fract Mech 2006;73:2642-61.

[17] da Silva LFM, Carbas RJC, Gritchlow GW, Figueiredo MAV, Brown K. Effect of material, geometry, surface treatment and environment on the shear strength of single lap joints. Int J Adhes Adhes 2009;29:621-32.

[18] da Silva LFM, Banea MD. Mechanical characterization of flexible adhesives. J Adhes 2009;85:261-85.

[19] da Silva LFM, Gritchlow GW, Figueiredo MAV. Parametric study of adhesively bonded joints. J Adhes Sci Technol 2008;22:1477-94.

[20] De Morais AB, Pereira AB, Teixeira JP, Cavaleiro NC. Strenght of epoxy adhesive-bonded stainless-steel joints. Int J Adhes Adhes 2007;27:679-86.

[21] Davies P, Sohier L, Cognard J-Y, Bourmaud A, Choqueuse D, Rinnert E, Créac'hcadec R. Influence of adhesive bond line thickness on joint strength. Int J Adhes Adhes 2009;29:724-36. 